ACCEPted For publication in the Astronomical Journal

Preprint typeset using $\mathrm{LATE}_{\mathrm{E}} \mathrm{X}$ style emulateapj v. 5/2/11

\title{
TIME-RESOLVED SPECTROSCOPY OF THE POLAR EU CANCRI IN THE OPEN CLUSTER MESSIER 67
}

\author{
Kurtis A. Williams \\ Department of Physics \& Astronomy \\ Texas A\&M University - Commerce \\ P.O. Box 3011, Commerce, TX, USA, 75429 \\ STEve B. Howell \\ NASA Ames Research Center \\ P.O. Box 1, M/S 244-30, Moffett Field, CA 94035 \\ JAmes Liebert AND PAul S. Smith \\ Steward Observatory \\ University of Arizona, Tucson, AZ \\ ANDREA BELLINI \\ Space Telescope Science Institute \\ 3700 San Martin Drive, Baltimore, MD 21218 \\ Kate H. R. Rubin \\ Max-Planck-Institut für Astronomie \\ Königstuhl 17, 69117 Heidelberg, Germany \\ AND \\ Michael Bolte \\ UCO/Lick Observatory \\ University of California \\ 1156 High St., Santa Cruz, CA, USA, 95064 \\ Accepted for publication in the Astronomical Journal
}

\begin{abstract}
We present time-resolved spectroscopic and polarimetric observations of the AM Her system EU Cnc. EU Cnc is located near the core of the old open cluster Messier 67; new proper motion measurements indicate that EU Cnc is indeed a member of the star cluster, this system therefore is useful to constrain the formation and evolution of magnetic cataclysmic variables. The spectra exhibit two-component emission features with independent radial velocity variations as well as time-variable cyclotron emission indicating a magnetic field strength of $41 \mathrm{MG}$. The period of the radial velocity and cyclotron hump variations are consistent with the previously-known photometric period, and the spectroscopic flux variations are consistent in amplitude with previous photometric amplitude measurements. The secondary star is also detected in the spectrum. We also present polarimetric imaging measurements of EU Cnc that show a clear detection of polarization, and the degree of polarization drops below our detection threshold at phases when the cyclotron emission features are fading or not evident. The combined data are all consistent with the interpretation that EU Cnc is a low-state polar in the cluster Messier 67. The mass function of the system gives an estimate of the accretor mass of $M_{\mathrm{WD}} \geq 0.68 M_{\odot}$ with $M_{\mathrm{WD}} \approx 0.83 M_{\odot}$ for an average inclination. We are thus able to place a lower limit on the progenitor mass of the accreting WD of $\geq 1.43 M_{\odot}$.

Subject headings: white dwarfs - novae, cataclysmic variables - Stars: individual: EU Cnc - open clusters and associations: individual: Messier 67 - Accretion, accretion disks
\end{abstract}

\section{INTRODUCTION}

Cataclysmic variables (CVs) are interacting binary systems in which a white dwarf (WD) is accreting material from a low-mass companion star. If the WD has a sufficiently strong magnetic field, the formation of an accretion disk is inhibited, and material accretes directly onto one or more magnetic poles of the white dwarf. These binaries are known as AM Her systems or polars, after the high fraction of polarized light detected in the systems.

kurtis.williams@tamuc.edu
The identification of CVs in star clusters provides interesting constraints on the formation and evolution of the interacting system. As all members of an cluster are coeval, the total age of the system is known, as is the system's distance and metallicity. Further, if the mass and effective temperature of the white dwarf can be determined, then a limit on the white dwarf's progenitor mass can be derived via the same methods used to construct the initial-final mass relation (e.g., Williams et al. 2009). Because the white dwarf is likely re-heated to some extent by the ongoing accretion, the constraint on 
the progenitor mass would be strictly a lower limit.

CVs are quite common in globular clusters (e.g. Margon et al. 1981; Grindlay et al. 1995). The globular CV population tends to be centrally concentrated (e.g. Grindlav et al. 1995, 2001). This result is likely explained by the formation of tight binaries by stellar encounters in globular cluster cores (e.g. Podsiadlowski et al. 2002; Pooley et al. 2003). As such, globular cluster CVs are excellent tracers of a globular's dynamic history, but these CVs may not shed much light on the formation and evolution of CVs in the galactic field where stellar encounters are rare.

Open star clusters may therefore be a more useful laboratory for studying $\mathrm{CV}$ evolution. The stellar densities are far lower than in globular clusters, even in the cluster core, and dynamical simulations suggest that CV formation is not enhanced by the stellar encounters that do occur (Shara \& Hurlev 2002), though a small fraction of open cluster CVs may still be formed by stellar exchanges (Shara \& Hurley 2006). Open clusters also span a wide range of ages, metallicities, and stellar masses, raising the potential to study how these parameters impact CV formation and evolution more precisely than possible from studies of field CVs.

Unfortunately, the number of CVs in open clusters is small, and none are well-studied. In the ancient, dense, metal-rich open cluster NGC 6791, two spectroscopicallyconfirmed CVs are known (Kaluzny et al. 1997; Mochejska et al. 2003); de Marchi et al. (2007) identify a suspected third cluster $\mathrm{CV}$ based on photometric properties and conclude that all three CVs are likely cluster members. One CV, EU Cnc, is known in the open cluster M67 and described in detail below. These four objects are the only confirmed cluster member $\mathrm{CVs}$, and due to the large distances of the clusters $\left[(m-M)_{V}=13.4\right.$ for NGC 6791 and $(m-M)_{V}=9.97$ for M67], spectroscopic studies of these CVs with the same precision and the same techniques (such as tomography) as current field $\mathrm{CV}$ studies require significant time on $8 \mathrm{~m}$-class telescopes and larger.

Other candidate open cluster CVs have been suggested: Mochejska et al. (2004, 2006) identify a CV in the field of the $\sim 2-3$ Gyr-old open cluster NGC 2158, though it may lie foreground to the cluster. One CV is identified photometrically in the field of the $3.5 \mathrm{Gyr}-$ old cluster NGC 6253, but no membership information is available (de Marchi et al. 2010). The rich open cluster M37 (age 550 Myr) has two CV candidates identified photometrically by Hartman et al. (2008). Finally, an X-ray source in the field of the cluster NGC 6819 (age $\sim 2-2.4$ Gyr) has properties consistent with CVs, but its true nature and cluster membership are unconfirmed (Gosnell et al. 2012). The Hyades contains at least one pre-CV, V471 Tau (e.g Vauclair 1972), but no actively accreting systems.

\subsection{EU Cancri}

EU Cnc was detected as variable object in the field of the old open star cluster Messier 67 by Gilliland et al. (1991), who identified it as a likely AM Her system based on the similarities of its light curve to that of VV Pup. They determined EU Cnc has a photometric period of $2.091 \pm 0.002 \mathrm{hr}$ with variations through a $\mathrm{CuSO}_{4}(U$ band) filter of 0.6 mag. Belloni et al. (1993) detected an X-ray source coincident with the opticallyvariable source; its very soft $\mathrm{X}$-ray hardness ratio is typical for AM Her systems in the ROSAT bands. The AM Her nature of EU Cnc was confirmed by Pasquini et al. (1994), who obtained three 75-minute optical spectra of the source. These spectra exhibit cyclotron humps and radial-velocity variable emission lines of $\mathrm{H}, \mathrm{He} \mathrm{I}, \mathrm{He}$ II, and Fe II. Under the assumption that EU Cnc is in the star cluster, Pasquini et al. (1994) conclude that the absolute optical magnitude and X-ray luminosity of EU Cnc are typical for low-state AM Her systems.

Subsequent X-ray studies by Belloni et al. (1998) using ROSAT detected $100 \%$ modulation of the soft X-ray flux with a period equal to the optical period, indicative of accretion onto a single magnetic pole. Chandra observations by van den Berg et al. (2004) detected hard X-ray emission from EU Cnc, again typical for AM Her systems and likely due to shocks in the accretion flow.

More recent time-series photometry from Nair et al. (2005) again detected high-amplitude optical modulation, with $V$-magnitudes varying from 21.6 to $20.3 \mathrm{mag}$ at the same $2.09 \mathrm{hr}$ period of Gilliland et al. (1991). The variation amplitude was about 30\% larger than in 1991 , with that difference likely due to different filters used in the two studies, $V$ containing a large cyclotron modulation.

Based on the body of work on EU Cnc, Nair et al. (2005) point out an interesting conundrum. The highamplitude optical variability is typical for AM Her systems in a high accretion state, while the X-ray luminosity of EU Cnc, under the assumption it is a member of M6\%, is typical of magnetic CVs in a low accretion state.

In this paper, we present time-resolved spectroscopy of EU Cnc obtained serendipitously with the 10-m Keck telescope, as well as the first polarimetric measurements of this system. After discussing the observed phenomenology, we revisit the issue of EU Cnc's cluster membership. We will show that the optical variability is almost entirely due to cyclotron emission changing throughout the orbit and that the optical spectrum is typical of a low accretion state polar. We detect the secondary star in the spectrum and, along with the other evidence, we show that EU Cnc is a member of the M67 open cluster.

\section{TIME-RESOLVED SPECTROSCOPY}

\subsection{Observations and Data Reduction}

We targeted EU Cnc serendipitously as part of a program to obtain high signal-to-noise spectroscopy of WDs in Messier 67. We obtained observations on UT 2007 Jan 19 with the low-resolution imaging spectrometer (LRIS) on the Keck I telescope (Oke et al. 1995; Steidel et al. 2004). We obtained simultaneous spectra with both blue and red sides of the spectrograph through a multiobject slit mask with slitlet widths of 1 ". 0 . On the blue side, we used the 400 lines $\mathrm{mm}^{-1}$ grism blazed at $3400 \AA$ for a resulting spectral resolution of $\approx 7 \AA$ FWHM. On the red side of the spectrograph, we used the D560 dichroic and the 600 grooves $\mathrm{mm}^{-1}$ grating blazed at $7500 \AA$, for a resulting spectral resolution of $4.8 \AA$ FWHM. The total spectral coverage ranged from $7400 \AA$ blueward to the UV atmospheric cutoff.

The weather was nearly photometric through the en- 


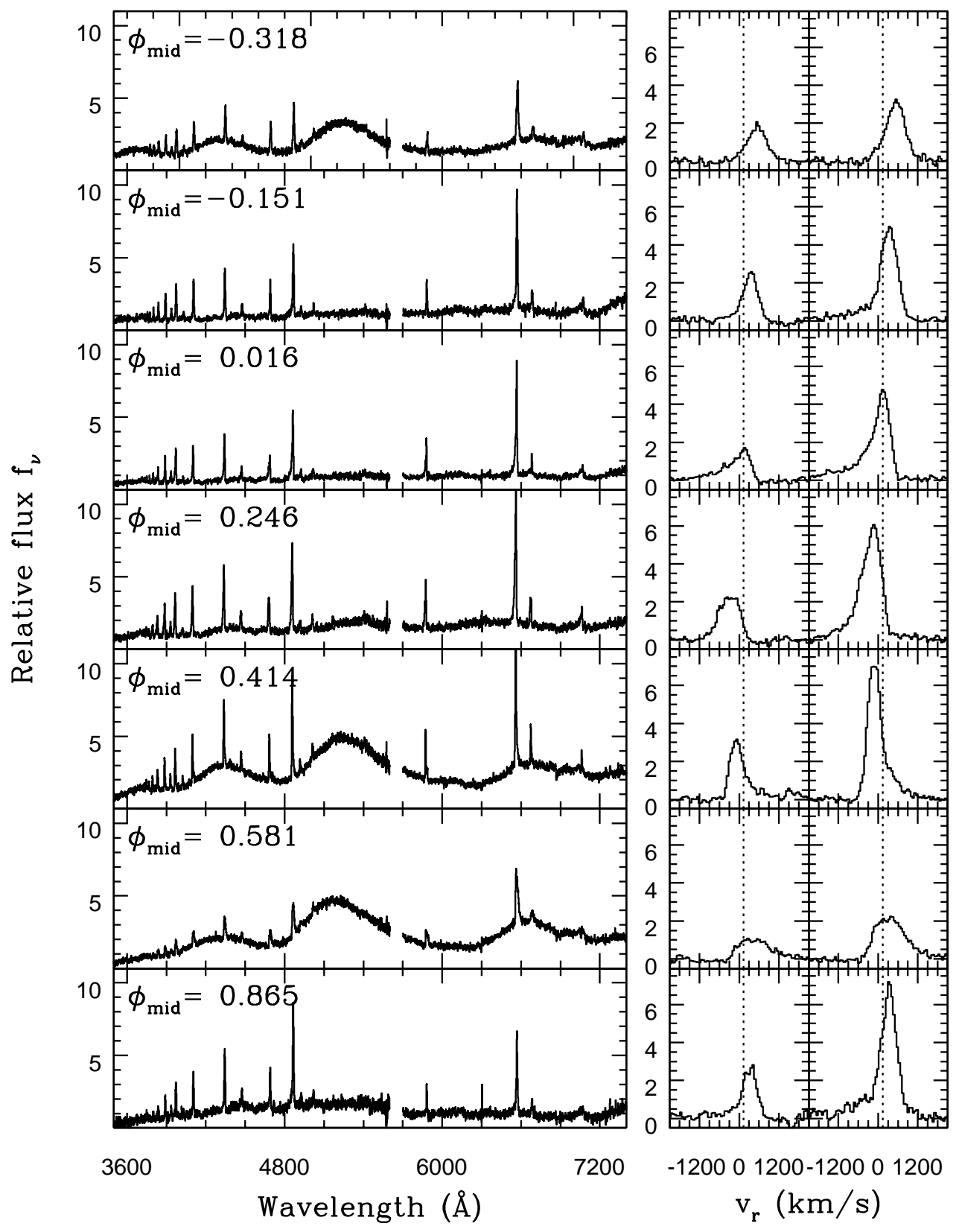

Figure 1. Time-ordered spectroscopy of EU Cnc. The left panels contain the entire spectrum; the gap at $\lambda \approx 5650 \AA$ is the gap between the blue and red arms of the spectrograph. The right panels contain close-ups of the $\lambda 4686 \mathrm{He}$ II line (left) and $\mathrm{H} \beta$ line (right); the radial velocity variations and variable line asymmetries are clearly visible. Phase $\phi=0$ indicates the negative zero crossing of the narrow-line component of the emission lines.

tire observation, and seeing was moderate at 0".9 FWHM. Seven exposures, each of twenty-minute integration, were taken over a $\sim 2.5 \mathrm{hr}$ period, with two short breaks for mask re-alignment. During the final exposure, the flux dropped dramatically for most stars, likely indicating that the mask was slightly misaligned.

These observations were taken prior to the installation of the LRIS atmospheric dispersion corrector, and due to constraints in slit mask design, the slitlets were oriented nearly perpendicular to the parallactic angle. We attempted to minimize the effects of atmospheric dispersion by using a blue filter in the guider camera, but diminution of the UV light is severe at higher airmasses.

We reduced the data using the onedspec and twodspec packages in IRAF. Overscan regions were used to determine and remove the amplifier bias. Flat-fielding on the blue side data was accomplished using a piecewisesmooth response function as described in Williams et al. (2009) to eliminate ringing due to a sharp inflection point in the flat field at $\approx 4200 \AA$. Cosmic rays were removed 
from each two-dimensional spectrum using the L.A. Cosmic Laplacian cosmic ray rejection routine van Dokkum 2001). Wavelength solutions on the blue side were derived from $\mathrm{Hg}, \mathrm{Cd}$, and $\mathrm{Zn}$ arclamp spectra; on the red side, Ne and Ar arclamp spectra were used. These calibrations were obtained prior to the final mask realignment.

Relative flux calibration was obtained using $1^{\prime \prime}$-wide long-slit spectroscopy of the spectrophotometric standards G191-B2B and G138-31 taken at parallactic angle near the start of the night. No attempt was made to obtain absolute spectrophotometric calibrations.

\subsection{Spectral Phenomenology}

The time-series spectra are shown in Figure 11 Qualitatively, the spectra appear fairly typical for AM Hertype systems in a low accretion state, similar to those observed for HU Aqr (Glenn et al. 1994). Cyclotron humps are visible and variable in strength. Emission lines of $\mathrm{H}$ and $\mathrm{He}$ are observed and have at least two components, a narrow (unresolved) component and a broad component. The emission lines have variable radial velocities, and the radial velocities of the two line components are not in phase. This type of emission line component behavior is often seen in polars, for example VV Pup (Mason et al. 2008) whereby the narrow line component phases with the motion of the secondary star (Mason et al. 2008; Howell et al. 2008). The time scales for both the cyclotron hump variations and emission line variations appear to be consistent with the known photometric period, though additional time-series spectra would be required to prove these are identical. We now quantify these phenomena.

We estimate the strength of the magnetic field using the spacing of the cyclotron humps in the optical spectra. The locations of the peaks are estimated by fitting a 12th-order polynomial to the continuum, excluding the obvious emission lines. We use equation 54 from Wickramasinghe \& Ferrario (2000) to get a magnetic field strength of $B \approx 41 \mathrm{MG}$. We do not see Zeeman split hydrogen absorption lines in the spectrum corresponding to this magnetic field strength, but later we will see that they are likely filled in by the red continuum contribution of the secondary star.

\subsubsection{Radial Velocity}

Because these data were not obtained with the goal of obtaining precision radial velocities, and because the flexure in the spectrograph was significant over the course of the observations, it was not possible to obtain absolute radial velocities. Attempts to use night sky emission lines as velocity zero points failed due to the lack of night sky lines in data from the blue arm of the spectrograph, where the majority of the CV's emission lines were located.

Instead, relative radial velocities were obtained as follows. First, the continuum was fit with a high-order polynomial and removed from each spectrum. The radial velocity of the narrow component of the emission lines was determined by autocorrelation of each spectrum with that of an observation with an exposure midpoint close to zero phase. This determination involved some iterative bootstrapping with the radial velocity fitting

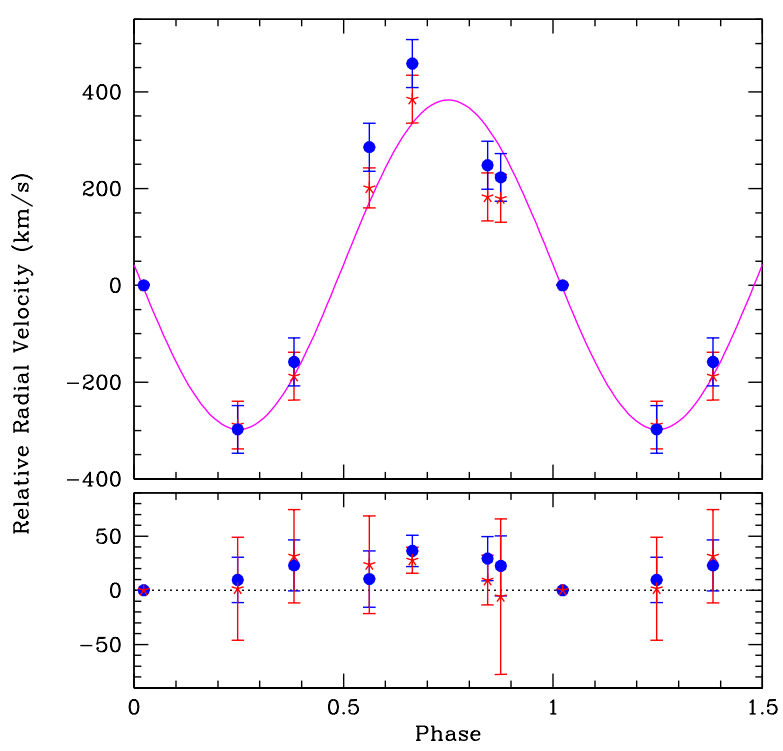

Figure 2. Radial velocity curve for EU Cnc (top panel) and a G-type star in a neighboring slitlet (bottom panel). Filled circles (blue in the online color version)indicate relative radial velocities from emission lines on the blue arm of the spectra; stars (red in the online version) indicate data from the red arm of the spectrograph. The solid curve (magenta in the online version) is the best-fitting sine wave.

described further below. The resulting radial velocities (relative to the observations closest to zero phase) are given in Table 1 and shown in Figure 2. The radial velocities at $\phi \approx 0.59$ are likely measuring different motion than the other points, as the narrow component of the emission lines disappears during these observations.

As a cross-check, we used the same method to determine the radial velocity of a G-type star targeted in a neighboring slitlet. The radial velocity of this star should be constant over the set of observations, and although a slight positive velocity offset may exist, it is of low amplitude compared with the variations observed in the CV.

The phase and amplitude of the radial velocity variations were determined by fitting a function of the form

$$
v_{r e l}=K \sin \left[2 \pi\left(\phi-\phi_{0}+0.5\right)\right]+v_{0}
$$

to the radial velocity data. The period was fixed to the known optical period of 2.091 hours, and the phase shift $\phi_{0}$, amplitude $K$, and the relative velocity zeropoint $v_{0}$ were allowed to vary. The best-fit values are $K=340 \pm 20 \mathrm{~km} \mathrm{~s}^{-1}$ and $v_{0}=+43 \pm 37 \mathrm{~km} \mathrm{~s}^{-1}$. We emphasize that this velocity zero point is a velocity relative to the spectra used as our zero velocity, which were selected since they are the closest data to a phase of zero. Based on the detailed observation of narrow line components in the polars VV Pup (Mason et al. 2008) and EF Eri (Howell et al. 2008), mapping to the motion of the secondary star, the typical orbital phase 0.0 used for cataclysmic variables would occur near phase 0.5 as shown in Figures 1 and 2. However, we do not have sufficient data here (e.g., a velocity curve of the photosphere of the secondary star) to state this fact with absolute certainty. As these velocities are not absolute radial velocities, they cannot be used for cluster membership determination or 
Table 1

Radial velocities of EU Cnc and an unrelated neighboring G-type star

\begin{tabular}{|c|c|c|c|c|c|c|}
\hline $\begin{array}{l}\text { Obs. Midpt } \\
\text { (MJD) }\end{array}$ & Phase & $\begin{array}{l}\text { Spectrograph } \\
\text { Arm }\end{array}$ & $\begin{array}{c}v_{\mathrm{EU} \mathrm{Cnc,rel}} \\
\left(\mathrm{km} \mathrm{s}^{-1}\right)\end{array}$ & $\begin{array}{c}\sigma\left(v_{\mathrm{EU} \mathrm{Cnc,rel}}\right) \\
\left(\mathrm{km} \mathrm{s}^{-1}\right)\end{array}$ & $\begin{array}{c}v_{*, \text { rel }} \\
\left(\mathrm{km} \mathrm{s}^{-1}\right)\end{array}$ & $\begin{array}{r}\sigma\left(v_{*, \text { rel }}\right) \\
\left(\mathrm{km} \mathrm{s}^{-1}\right)\end{array}$ \\
\hline 54119.51236 & 0.682 & Red & 384.9 & 13.8 & 27.8 & 5.2 \\
\hline 54119.52732 & 0.854 & Red & 183.0 & 23.2 & 9.4 & 20.8 \\
\hline 54119.54231 & 0.026 & Red & 0.0 & 0.0 & 0.0 & 0.0 \\
\hline 54119.56151 & 0.246 & Red & -288.7 & 27.6 & 1.5 & 46.9 \\
\hline 54119.57651 & 0.418 & Red & -187.5 & 28.1 & 31.5 & 42.4 \\
\hline 54119.59146 & 0.590 & Red & 201.4 & 25.6 & 23.8 & 44.3 \\
\hline 54119.61542 & 0.865 & Red & 179.7 & 23.5 & -5.9 & 71.1 \\
\hline 54119.51239 & 0.682 & Blue & 458.5 & 17.9 & 36.3 & 11.8 \\
\hline 54119.52689 & 0.849 & Blue & 248.3 & 27.5 & 29.3 & 18.8 \\
\hline 54119.54145 & 0.016 & Blue & 0.0 & 0.0 & 0.0 & 0.0 \\
\hline 54119.56153 & 0.246 & Blue & -297.7 & 30.5 & 9.7 & 19.9 \\
\hline 54119.57613 & 0.414 & Blue & -158.2 & 24.9 & 22.9 & 21.2 \\
\hline 54119.59067 & 0.581 & Blue & 285.7 & 33.6 & 10.5 & 22.7 \\
\hline 54119.61545 & 0.865 & Blue & 223.2 & 35.2 & 22.6 & 25.0 \\
\hline
\end{tabular}

Note. - Phase $\phi=0$ indicates the negative zero crossing of the narrow-line component of the emission lines; velocities are relative to the exposure closeest to $\phi=0$.

rejection.

Qualitatively, the sine curve is not a superb fit to the data, especially for phases between 0.5 and 1 . We note that, at these phases, the narrow component of the lines is relatively weak and the broad component strong. In fact, at phase $\phi=0.59$, the narrow component of the lines is not visible. These velocity measurements are likely non-Keplerian streaming motion. However, the excellent fit for the points with phases between 0 and 0.5, where the emission lines are dominated by the narrow component, suggests that our fit amplitude and phase shift are not unreasonable.

\subsubsection{Photometric Variability}

As mentioned above, absolute spectrophotometry was not a goal of our observations; slit losses as a function of wavelength could be significant and time-variable due to both seeing and atmospheric dispersion effects. Even so, it would be instructive to compare photometry derived from our spectroscopy with previous photometric monitoring of this system. We therefore calculate broadband photometry by folding our (relative) flux-calibrated spectra through filter response functions. Due to the significant loss of UV light in the later exposures, we restrict our analysis to the $g$-band.

In order to correct for slit losses and variable atmospheric absorption, we perform the same calculation for a K-type star targeted in a different slitlet on the same mask (note that this is not the same star used for radial velocity comparisons in 92.2 .1$)$. The star is located at $\alpha(\mathrm{J} 2000)=8^{\mathrm{h}} 51^{\mathrm{m}} 35.46, \delta(\mathrm{J} 2000)=11^{\circ} 50^{\prime} 19^{\prime \prime} .1$, and, in our photometry (Williams et al, in preparation), has $g=20.324 \pm 0.032$. This star also appears in SDSS DR7, with PSF magnitude $g=20.263 \pm 0.038$. Due to the higher precision of our data, we adopt our photometry for this star.

For each exposure, the calibrated spectra of both EU Cnc and the comparison star are folded through the $g$ band filter response function using the Synphot synthetic photometry package in STSDAS. All magnitudes are calculated as AB magnitudes. Zero-point offsets in $g$ are calculated from the comparison star; these offsets are then applied to the calculated magnitudes for EU Cnc. The
Table 2

Time Series Broad-band Photometry of EU Cnc

\begin{tabular}{cccc}
\hline \hline $\begin{array}{c}\text { Midpoint Obs. } \\
\text { (HJD) }\end{array}$ & $\begin{array}{c}g_{*, \text { meas }}{ }^{\mathrm{a}, \mathrm{b}} \\
(\mathrm{mag})\end{array}$ & $\begin{array}{c}g_{\text {EU Cnc, meas }}{ }^{\mathrm{a}} \\
(\mathrm{mag})\end{array}$ & $\begin{array}{c}g_{\text {EU Cnc, corr }}{ }^{\mathrm{c}} \\
(\mathrm{mag})\end{array}$ \\
\hline 2454120.01789 & 20.563 & 20.806 & 20.567 \\
2454120.03239 & 20.676 & 21.601 & 21.249 \\
2454120.04695 & 20.480 & 21.672 & 21.516 \\
2454120.06703 & 20.703 & 21.266 & 20.887 \\
2454120.08163 & 20.614 & 20.440 & 20.150 \\
2454120.09617 & 20.467 & 20.429 & 20.286 \\
2454120.12095 & 21.147 & 21.706 & 20.883 \\
& & & \\
\hline
\end{tabular}

${ }^{a}$ Measured flux folded through $g$ filter response

b Instrumental magnitude of the neighboring $\mathrm{K}$ star

${ }^{c}$ EU Cnc photometry corrected to standard system

results are included in Table 2. The errors in this photometry are uncertain. The random errors due to photon shot noise in the source and sky are small, $\leq 0.003 \mathrm{mag}$. However, systematic errors such as differential slit losses likely dominate the photometric uncertainty. The magnitude of the error in absolute photometry is at least $0.03 \mathrm{mag}$ (the error in the broadband photometry of the comparison star).

We compare our derived photometry with the timeseries photometry of Nair et al. (2005). These published data were taken in $V$; we converted these magnitudes to $g$ using the Population I transformation equations in Table 4 of Jordi et al. (2006) and the $B-V=0.41$ color of EU Cnc from Gilliland et al. (1991). As these transformations are for single stars, and as the color of EU Cnc is likely changing as a function of phase, we emphasize that these transformations are meant to be illustrative only. Since the ephemeris of EU Cnc is not sufficiently well-determined to allow us to phase the data precisely, we added an arbitrary phase shift to the published photometry.

The results of this comparison are shown in Figure 3. With the exception of our observation at $\phi=0.865$, our corrected spectrophotometry and the published timeseries photometry agree very well in both shape and amplitude. 


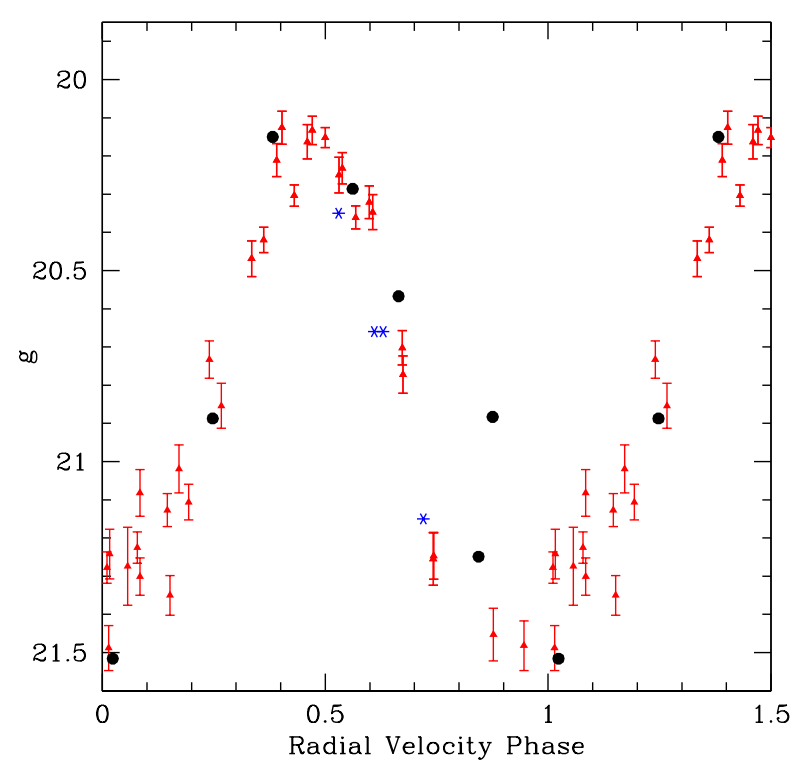

Figure 3. The light curve for EU Cnc. Large filled circles are the spectrophotometry data from this work; error bars are not included but are likely at least 0.03 mag. Filled triangles with error bars (red in the online version) are data from Nair et al. (2005), shifted in phase by an arbitrary offset. Stars (blue in the online version) are spectropolarimetric data form this work. The light curves are qualitatively nearly identical between these data sets, suggesting little change in the state of the polar between early 2004 and late 2007.

\subsection{Time-series Polarimetry}

Circular polarimetry of EU Cnc was obtained with the SPOL spectropolarimeter (Schmidt et al. 1992) on the 2.3-m Bok Telescope at Steward Observatory in 2007 December. The observations were taken in the imaging mode of the instrument using a Hoya HA30+Y48 filter combination, giving a broad bandpass of $\approx 4800-7000$ $\AA$. Image acquisition and data reduction follow those described in Smith et al. (2002), but modified as appropriate for circular polarimetry. A $\lambda / 4$ wave plate is used to convert incident circular polarization to linear and the Wollaston prism separates the light into two orthogonally polarized beams that are focused onto a CCD. Two separate reads of the CCD are made with the wave plate rotated through four positions to determine the circular $\mathrm{V}$ Stokes parameter. Two consecutive 10-minute exposures (150 s per wave plate position) were obtained on each of two nights. The sky was clear during the observations, but no attempt at absolute calibration was made because of the non-standard filter bandpass used. Photometric information was extracted using a circular aperture of radius $6^{\prime \prime}$.

The observing log and resulting circular polarization measurements $(V / I=v)$ are given in Table3. Also given are the background subtracted counts and the phase of the midpoint of each observation relative to first exposure on the first night assuming a photometric period of 2.091 $\mathrm{h}$. There is a significant detection of polarization in the first exposure, with $v=-12.7 \pm 1.8 \%$. The degree of polarization and the total flux both drop significantly in the second exposure $(v=-6.3 \pm 2.3 \%)$. The third exposure, taken two nights later, is at nearly the same phase as the second exposure. The total flux is slightly lower and there is no significant detection of polarization $(v=-2.8 \pm 2.6)$; the final exposure is the faintest of the four and has no significant polarization.

As these observations were taken 11 months after the spectroscopy, and as the ephemeris of EU Cnc is not sufficiently well known, the spectroscopic phase of these observations cannot be calculated - the accumulated uncertainty in phase given the $0.002 \mathrm{hr}$ uncertainty in the photometric period of Gilliland et al. (1991) is $\approx 7.5$ cycles. However, from the total counts in the polarimetry measurements, we know that these observations were taken on the declining portion of the light curve. We therefore calculate relative magnitudes from the total intensity measurements in Table 3 and add an arbitrary magnitude zero point and phase shift to place these points in the folded light curve of Figure 3 .

From this exercise, we see that the rate of decline in the observed intensity agrees with that observed in the timeseries photometry of Nair et al. (2005) and in our spectrophotometry, indicating that the spectroscopic phase of the first polarimetric observations was $\phi \approx 0.5$ to 0.65 . Comparison with the time-series spectra in Figure 1 shows that this corresponds to a phase when the cyclotron humps are dominant in the spectrum; these humps then vanish by a phase $\phi=0.85$. This is consistent with the strong degree of polarization observed in the first exposure, and the weaker/insignificant polarization in the other polarimetry exposures.

In summary, we detect significant polarization from EU Cnc at a phase likely corresponding to strong spectroscopic cyclotron emission features, and the degree of polarization decreased below our detection threshold at phases when the cyclotron features were fading or not evident in the spectrum. This proves that the features are indeed cyclotron emission. From a semantic point of view, this detection of significant polarization also confirms that we can use the moniker "polar" to refer to EU Cnc.

\section{DISCUSSION}

\subsection{Cluster Membership}

EU Cnc is projected $\approx 1.7$ away from the cluster center defined by Montgomerv et al. (1993) (0.4 pc at the cluster distance), well within the observed core radius of $\approx 4.5$ (e.g., Tinsley \& King 1976; Bonatto \& Bica 2003). However, this proximity alone is not sufficient evidence of cluster membership.

Bellini et al. (2010) present proper-motion data for white dwarfs in M67, precise to $V \approx 26$, obtained from multiple-epoch imaging from the Canada-France-Hawaii Telescope and the Large Binocular Telescope. Figure 4 shows the vector-point diagram of objects within $20^{\prime}$ from the cluster center (right panel). The circle indicates the proper motion membership cut adopted by Bellini et al. (2010); the error bars show the displacement uncertainty for EU Cnc, solidly inside the membership circle. In addition, the proper-motion selected color magnitude diagram (left panel) shows that EU Cnc lies on the WD cooling sequence of M67.

Astrometry performed on EU Cnc and 3 encircling brighter known member stars, using the POSS I and II plates as well as a deep, 0 ". 4 seeing image obtained by us 
Table 3

Polarimetric Observations of EU Cnc

\begin{tabular}{|c|c|c|c|c|c|}
\hline $\begin{array}{c}\text { Observation Date } \\
\text { (UT) }\end{array}$ & $\begin{array}{l}\text { Obs. Time } \\
\text { (UT) }\end{array}$ & $\begin{array}{c}\text { Relative Phase }{ }^{\mathrm{a}} \\
(\Delta \Phi)\end{array}$ & $\begin{array}{l}\text { Counts } \\
\text { (ADU) }\end{array}$ & $\begin{array}{l}V / I \\
(\%) \\
\end{array}$ & $\begin{array}{c}\sigma(V / I) \\
(\%)\end{array}$ \\
\hline 2007 December 14 & $10: 28$ & & 74147 & -12.7 & 1.8 \\
\hline 2007 December 14 & $10: 39$ & 0.08 & 55485 & -6.3 & 2.3 \\
\hline 2007 December 16 & 08:41 & 0.10 & 48303 & -2.8 & 2.6 \\
\hline 2007 December 16 & $08: 52$ & 0.19 & 30698 & 4.6 & 4.0 \\
\hline
\end{tabular}

${ }^{a}$ Phase relative to first polarimetric observation

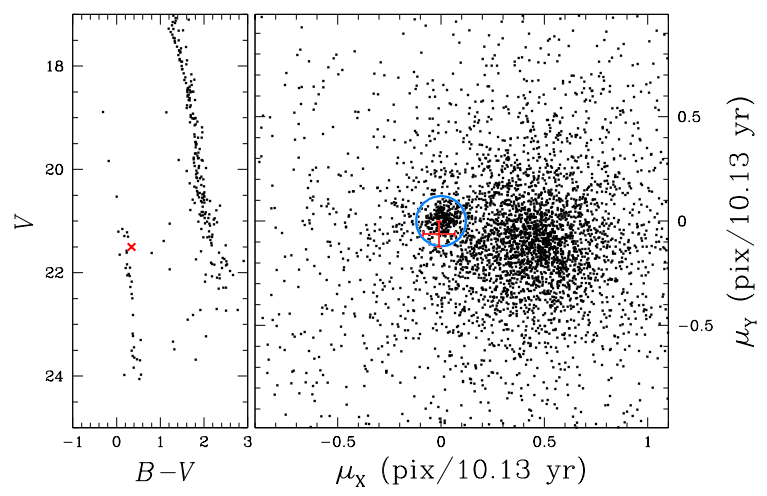

Figure 4. The proper-motion selected color-magnitude diagram (CMD, left) for M67 and the vector point diagram (VPD, right) for all stars in the field as determined by Bellini et al. (2010). The cross (red in the online version) in the CMD indicates the location of EU Cnc. The circle (blue in the online version) in the VPD indicates the selection criteria Bellini et al. used to select cluster members; the error bars (red in the online version) show the location and uncertainty in the proper motion of EU Cnc. The qualitative data are convincing that $\mathrm{EU} \mathrm{Cnc}$ is a proper-motion member of the cluster.

at the Kitt Peak WIYN telescope in 2007, show that the polar's location relative to the other three stars changes by $0 " .3 \pm 0 . .5$ over the 60 year interval. This is a similar uncertainty to that for each of the brighter stars relative to each other. We therefore feel confident that EU Cnc is a member of the M67 star cluster.

\subsection{A low-state polar in $M 67$}

Nair et al. (2005), suggest that the luminosity of EU Cnc is lower than other high state polars and fully consistent with the luminosity of low-state polars in globular clusters; the distances to field magnetic CVs are too uncertain to allow for such a precise comparison. The spectra obtained in this study, as well as those prior, show the clear indications of a low state polar: a steep Balmer decrement (compared to a flat decrement and Balmer jump in emission in high states) and the separable narrow and broad emission line components. Warner (1999) examine the high-low state range of polars and notes that the magnitude difference is typically near 3-4 for a 2 hour polar. Additionally, low state polars have absolute magnitudes near 11-12 in $V$ compared with 8-9 in $V$ when in a high state. If EU Cnc were in a high state with its observed apparent $V$ magnitude, it would need to reside at a distance of near 3100 pc. However, in its low state and $V \sim 21$, we find it to be at a distance of approximately $850 \mathrm{pc}$ or the same distance as M67 (Sarajedini et al. 2009).

Additionally, we detect the secondary star in the op-

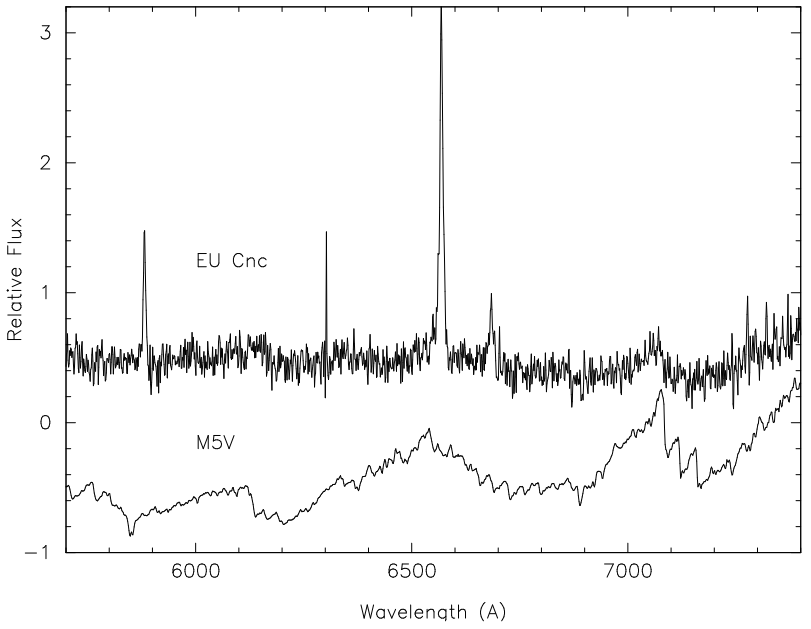

Figure 5. Red spectrum from phase 0.865 in Fig. 1 along with a scaled M5V spectrum taken from the Jacoby Atlas (star 57, Jacoby et al. 1984). The M5V star is scaled 1.4 magnitudes fainter than the stellar continuum, approximating the level of contribution is provides to the red end of the spectrum of EU Cnc.

tical spectrum of EU Cnc (see the late phase red spectra in Fig. 1 and Fig. 5y noting the $\mathrm{TiO}$ absorption bands chopping into the spectrum at the characteristic locations near $6800 \AA$ and $7100 \AA$. The shape and relative amplitude of the $\mathrm{TiO}$ humps are a good match to a M5V star which is also the expected spectral type of the secondary star in EU Cnc (see below). Noting the dilution of the $\mathrm{TiO}$ bands compared to a single M5V star, we determine that the secondary star contributes $20-25 \%$ of the continuum redward of $6000 \AA$. This estimate suggests that the M5V secondary star will have an apparent magnitude near 22.5 and, for an its $\mathrm{M}_{V}=12.6$, yields a distance to EU Cnc of 832 pc, again the same as M67. The $\mathrm{M}$ star continuum is also the likely cause of filling in the Zeeman spilt $\mathrm{H} \alpha$ absorption components.

\subsection{The masses of the white dwarf accretor and its progenitor star}

An advantage of finding a magnetic $\mathrm{CV}$ in an open star cluster is that numerous constraints can be placed on the progenitor system. As EU Cnc is a member of Messier 67 , its initial metallicity and total evolutionary age are identical to the cluster's characteristics. With some reasonable assumptions, we can go one step further to constrain the white dwarf's mass and cooling age, which we can then use to constrain the white dwarf progenitor's mass. We describe these steps in detail below, but note that this methodology has been used in numerous open cluster and field star studies to determine WD progen- 
itor masses (e.g. Liebert et al. 2005; Kalirai et al. 2008; Catalán et al. 2008; Williams et al. 2009; Dobbie et al. 2012)

WD masses are often determined through model atmosphere fits of the WD spectrum, but absorption lines from the white dwarf photosphere are not convincingly evident in our data, being filled in and misshaped by the emission. However, we note that Liebert \& Stockman (1985) and Mason et al. (2008) determined that the "center of mass" for the narrow emission lines originates between $L_{1}$ and the center of mass of the donor, that is they approximate the center of mass of the secondary star. We therefore assume that the narrow component of the emission lines originates from the center of mass of the secondary star and use our velocity amplitude to determine the mass function of the system and to estimate the mass of the white dwarf, $M_{\mathrm{WD}}$.

The mass function $f(M)$ of the system is

$$
f(M)=\frac{\left(M_{\mathrm{WD}} \sin i\right)^{3}}{\left(M_{\mathrm{WD}}+M_{2}\right)^{2}}=\frac{P K^{3}\left(1-e^{2}\right)^{3 / 2}}{2 \pi G}
$$

where $M_{2}$ is the mass of the donor star. Assuming a circular orbit $(e=0)$ with a period $P=2.091 \mathrm{hr}$ and the velocity amplitude $K=340 \mathrm{~km} \mathrm{~s}^{-1}$, from Eq. 1 we find $f(M)=0.356 M_{\odot}$.

Since we do not see eclipses, we can constrain the inclination to be $\lesssim 74^{\circ}$ (e.g. Warner 1995). If we assume $M_{\mathrm{WD}}>>M_{2}$, we therefore find that $M_{\mathrm{WD}} \geq 0.40 M_{\odot}$. If instead we assume an average value of $\left\langle\sin ^{3} i\right\rangle=0.679$ (e.g. Trimble 1974), then we find $M_{\mathrm{WD}}=0.52 M_{\odot}$.

However, the mass of the donor star is not negligible. Howell et al. (2001) present detailed evolution models for the secondary stars in cataclysmic variables and note that for those systems with orbital periods below the period gap $(<2.5 \mathrm{hr})$, the secondary stars follow the normal main sequence mass-radius relationship. We therefore expect the mass of the secondary star in EU Cnc to be $M_{2}=0.21 M_{\odot}$ with a radius of $R_{2}=0.22 R_{\odot}$. These value are roughly those of an M5V star. Adopting this mass for the secondary star, we find $M_{\mathrm{WD}} \gtrsim 0.68 M_{\odot}\left(\right.$ since $\left.i \lesssim 74^{\circ}\right)$ and $M_{\mathrm{WD}}=0.83 M_{\odot}$ for $\left\langle\sin ^{3} i>=0.679\right.$.

We note that these masses assume that our velocity amplitude is correct. As noted in 2.2.1. our value of $K$ assumes that our velocity amplitude is resolving the narrow component of the emission lines and that the narrow lines trace the center of mass of the secondary star. Higher spectral resolutions would be necessary to test the first assumption, and so we do not have good constraints on the errors or our confidence limits on the white dwarf mass. However, the WD mass estimates are similar to or higher than the spectroscopic masses of young member WDs in M67 $\left(M=0.5 M_{\odot}\right.$ to $0.6 M_{\odot}$, Williams et al. in preparation). Further, through a lucky coincidence, our ignorance of the WD mass does not significantly impact estimates of the WD's progenitor mass. We therefore proceed (perhaps quixotically) to constrain the WD's progenitor mass.

To constrain the progenitor mass, we use the methodology of Williams et al. (2009). To summarize, we use the effective temperature and mass of the WD to determine its cooling age (the elapsed time since the WD emerged from the AGB progenitor star). This cooling age is subtracted from the cluster age to get the progenitor star's nuclear lifetime; stellar evolutionary models are then used to infer the progenitor star mass.

The WD mass estimates are sufficiently high to conclude that the WD has a carbon-oxygen core, indicating that the common envelope phase for the progenitor system did not occur before helium ignition in the WD progenitor. Since the AGB phase of stellar evolution is relatively short compared to the WD cooling times and progenitor nuclear lifetimes we estimate for this star, we assume that any effect of the common envelope phase on the calculation of the progenitor nuclear lifetime is minimal.

To estimate the effective temperature of the WD, we assume that the minimum luminosity of the system is due to solely to the combined light of the M5V secondary and the WD photosphere, and we assume that the WD has not been heated by the ongoing accretion. Under these assumptions, the WD has $M_{V}=12.5$. We then interpolate WD photometric evolutionary tables provided online by $\mathrm{P}$. Bergeron 1 and computed from color and model calculations of Holberg \& Bergeron (2006), Kowalski \& Saumon (2006), Tremblay et al. (2011), and Bergeron et al. (2011) to find the WD's cooling age as a function of mass at which its luminosity is equal to the observed minimum luminosity of the CV. Despite the uncertainty in the WD mass, the cooling age of the WD is relatively insensitive to its mass, at least over the range of $0.5 M_{\odot}$ to $1.0 M_{\odot}$, at $\log \tau_{\text {cool }}=8.86$ to 8.95 . If the WD has experienced significant heating, or if the minimum luminosity is significantly contaminated by light from the accretion stream and/or the accretion column, then its actual cooling age will be older than these calculations. This means that our derived progenitor mass is a lower limit on the WD's actual progenitor mass.

We now calculate the WD progenitor mass. We adopt an age of M67 of 3.5 Gyr to $4.5 \mathrm{Gyr}$, and a cluster metallicity of $Z_{M 67} \approx Z_{\odot}$ and the calculated primordial value of $Z_{\odot}=0.0142$ (Grevesse et al. 2010). Since the lower limit on the WD cooling age is well-constrained, we obtain a fairly precise lower limit on the progenitor mass of $M_{\mathrm{WD} \text {,init }}=1.43 M_{\odot} \pm 0.07 M_{\odot}$, with the majority of the uncertainty due to the uncertainty in the age of M67. For comparison, the current main sequence turnoff mass (using the same models and inputs) is $1.35 M_{\odot}$.

If we relax the assumption of no heating of the WD, then the WD's cooling age (time since its emergence from the red giant progenitor) could be significantly longer; this would imply a larger progenitor mass. Therefore, we can only constrain the WD progenitor mass calculated above to be a lower limit; i.e. $M_{\mathrm{WD} \text {,init }} \geq 1.43 M_{\odot}$.

\section{CONCLUSIONS}

We have presented new observations of the polar EU Cnc. In particular, proper motion studies strongly indicate that this system is a bona-fide member of the old open star cluster M67, which provides strong constraints on EU Cnc's total age, metallicity, and progenitor mass. Using phase-resolved optical spectra and polarimetry, we have shown that EU Cnc exhibits all the properties of a low state polar. Both assumed "best fit" polar parameters as well as standard values for the donor star lead to

\footnotetext{
1 http://www . astro.umontreal.ca/ \{\}bergeron/CoolingModels
} 
conclusions that are consistent with the system's membership in M67. EU Cnc is one of only four CVs confirmed to reside in open clusters and the only confirmed magnetic $\mathrm{CV}$ in an open cluster. More detailed photometric, polarimetric, and spectroscopic observations on large telescopes will be required to refine the system parameters and model the system with state-of-the-art analyses. However models of magnetic cataclysmic variable formation and evolution are not usually attempted and never well constrained; further study of EU Cnc will greatly aid in these endeavors.

K.A.W. is grateful for the financial support of National Science Foundation awards AST-0397492 and AST-0602288. The authors thank S. Kafka for discussions on this system and for providing the original data from Nair et al. (2005). We also thank G. Schmidt for providing time from his observing run to obtain the polarimetry in this paper, D. Wickramasinghe for assistance in measuring the magnetic field strength, and the anonymous referee for suggestions leading to improvement of this paper. The authors wish to recognize and acknowledge the very significant cultural role and reverence that the summit of Mauna Kea has always had within the indigenous Hawaiian community. We are most fortunate to have the opportunity to conduct observations from this mountain.

Facilities: Keck:I (LRIS-B), Bok (SPOL)

\section{REFERENCES}

Bellini, A., Bedin, L. R., Piotto, G., et al. 2010, A\&A, 513, A50 Belloni, T., Verbunt, F., \& Mathieu, R. D. 1998, A\&A, 339, 431 Belloni, T., Verbunt, F., \& Schmitt, J. H. M. M. 1993, A\&A, 269, 175

Bergeron, P., Wesemael, F., Dufour, P., et al. 2011, ApJ, 737, 28 Bonatto, C., \& Bica, E. 2003, A\&A, 405, 525

Catalán, S., Isern, J., García-Berro, E., et al. 2008, A\&A, 477, 213 de Marchi, F., Poretti, E., Montalto, M., Desidera, S., \& Piotto, G. 2010, A\&A, 509, A17

de Marchi, F., Poretti, E., Montalto, M., et al. 2007, A\&A, 471, 515

Dobbie, P. D., Day-Jones, A., Williams, K. A., et al. 2012, MNRAS, 423, 2815

Gilliland, R. L., Brown, T. M., Duncan, D. K., et al. 1991, AJ, 101,541

Glenn, J., Howell, S. B., Schmidt, G. D., et al. 1994, ApJ, 424, 967

Gosnell, N. M., Pooley, D., Geller, A. M., et al. 2012, ApJ, 745, 57

Grevesse, N., Asplund, M., Sauval, A. J., \& Scott, P. 2010, Ap\&SS, 328, 179

Grindlay, J. E., Cool, A. M., Callanan, P. J., et al. 1995, ApJ, 455, L47

Grindlay, J. E., Heinke, C. O., Edmonds, P. D., Murray, S. S., \& Cool, A. M. 2001, ApJ, 563, L53
Hartman, J. D., Gaudi, B. S., Holman, M. J., et al. 2008, ApJ, 675,1254

Holberg, J. B., \& Bergeron, P. 2006, AJ, 132, 1221

Howell, S. B., Harrison, T. E., Huber, M. E., et al. 2008, AJ, 136, 2541

Howell, S. B., Nelson, L. A., \& Rappaport, S. 2001, ApJ, 550, 897

Jacoby, G. H., Hunter, D. A., \& Christian, C. A. 1984, ApJS, 56, 257

Jordi, K., Grebel, E. K., \& Ammon, K. 2006, A\&A, 460, 339

Kalirai, J. S., Hansen, B. M. S., Kelson, D. D., et al. 2008, ApJ, 676,594

Kaluzny, J., Stanek, K. Z., Garnavich, P. M., \& Challis, P. 1997, ApJ, 491, 153

Kowalski, P. M., \& Saumon, D. 2006, ApJ, 651, L137

Liebert, J., \& Stockman, H. S. 1985, in Astrophysics and Space Science Library, Vol. 113, Cataclysmic Variables and Low-Mass X-ray Binaries, ed. D. Q. Lamb \& J. Patterson, 151-177

Liebert, J., Young, P. A., Arnett, D., Holberg, J. B., \& Williams, K. A. 2005, ApJ, 630, L69

Margon, B., Downes, R. A., \& Gunn, J. E. 1981, ApJ, 247, L89

Mason, E., Howell, S. B., Barman, T., Szkody, P., \& Wickramasinghe, D. 2008, A\&A, 490, 279

Mochejska, B. J., Stanek, K. Z., \& Kaluzny, J. 2003, AJ, 125, 3175

Mochejska, B. J., Stanek, K. Z., Sasselov, D. D., et al. 2004, AJ, 128,312

-. 2006, AJ, 131, 1090

Montgomery, K. A., Marschall, L. A., \& Janes, K. A. 1993, AJ, 106,181

Nair, P. H., Kafka, S., Honeycutt, R. K., \& Gilliland, R. L. 2005, Information Bulletin on Variable Stars, 5585, 1

Oke, J. B., Cohen, J. G., Carr, M., et al. 1995, PASP, 107, 375

Pasquini, L., Belloni, T., \& Abbott, T. M. C. 1994, A\&A, 290, L17

Podsiadlowski, P., Rappaport, S., \& Pfahl, E. D. 2002, ApJ, 565, 1107

Pooley, D., Lewin, W. H. G., Anderson, S. F., et al. 2003, ApJ, 591, L131

Sarajedini, A., Dotter, A., \& Kirkpatrick, A. 2009, ApJ, 698, 1872

Schmidt, G. D., Stockman, H. S., \& Smith, P. S. 1992, ApJ, 398, L57

Shara, M. M., \& Hurley, J. R. 2002, ApJ, 571, 830

-. 2006, ApJ, 646, 464

Steidel, C. C., Shapley, A. E., Pettini, M., et al. 2004, ApJ, 604, 534

Tinsley, B. M., \& King, I. R. 1976, AJ, 81, 835

Tremblay, P.-E., Bergeron, P., \& Gianninas, A. 2011, ApJ, 730, 128

Trimble, V. 1974, AJ, 79, 967

van den Berg, M., Tagliaferri, G., Belloni, T., \& Verbunt, F. 2004, A\&A, 418, 509

van Dokkum, P. G. 2001, PASP, 113, 1420

Vauclair, G. 1972, A\&A, 17, 437

Warner, B. 1995, Cataclysmic Variable Stars (Cambridge University Press)

Warner, B. 1999, in Astronomical Society of the Pacific Conference Series, Vol. 157, Annapolis Workshop on Magnetic Cataclysmic Variables, ed. C. Hellier \& K. Mukai, 63

Wickramasinghe, D. T., \& Ferrario, L. 2000, PASP, 112, 873

Williams, K. A., Bolte, M., \& Koester, D. 2009, ApJ, 693, 355 